\title{
Crucial role of the protein C pathway in governing microvascular inflammation in inflammatory bowel disease
}

\author{
Franco Scaldaferri,, 1,2 Miquel Sans, ${ }^{3}$ Stefania Vetrano, ${ }^{1}$ Cristina Graziani, ${ }^{2}$ Raimondo De Cristofaro, ${ }^{2}$ \\ Bruce Gerlitz, ${ }^{4}$ Alessandro Repici, ${ }^{1}$ Vincenzo Arena, ${ }^{5}$ Alberto Malesci, ${ }^{1}$ Julian Panes, ${ }^{3}$ \\ Brian W. Grinnell, ${ }^{4}$ and Silvio Danese ${ }^{1}$

\begin{abstract}
1Division of Gastroenterology, Istituto di Ricovero e Cura a Carattere Scientifico (IRCCS) Istituto Clinico Humanitas, Rozzano, Italy. ${ }^{2}$ Institute of Internal Medicine, Catholic University, Rome, Italy. ${ }^{3}$ Department of Gastroenterology, Hospital Clinic y Provincial, Barcelona, Spain. ${ }^{4}$ Biotechnology Discovery Research, Lilly Research Laboratories, Indianapolis, Indiana, USA. ${ }^{5}$ Department of Pathology, Catholic University, Rome, Italy.
\end{abstract}

\begin{abstract}
Endothelial protein C receptor (EPCR) and thrombomodulin (TM) are expressed at high levels in the resting microvasculature and convert protein $\mathrm{C}$ (PC) into its activated form, which is a potent anticoagulant and antiinflammatory molecule. Here we provide evidence that in Crohn disease (CD) and ulcerative colitis (UC), the 2 major forms of inflammatory bowel disease (IBD), there was loss of expression of endothelial EPCR and TM, which in turns caused impairment of PC activation by the inflamed mucosal microvasculature. In isolated human intestinal endothelial cells, administration of recombinant activated PC had a potent antiinflammatory effect, as demonstrated by downregulated cytokine-dependent cell adhesion molecule expression and chemokine production as well as inhibited leukocyte adhesion. In vivo, administration of activated PC was therapeutically effective in ameliorating experimental colitis as evidenced by reduced weight loss, disease activity index, and histological colitis scores as well as inhibited leukocyte adhesion to the inflamed intestinal vessels. The results suggest that the PC pathway represents a new system crucially involved in governing intestinal homeostasis mediated by the mucosal microvasculature. Restoring the PC pathway may represent a new therapeutic approach to suppress intestinal inflammation in IBD.
\end{abstract}

\section{Introduction}

The regulatory pathways involved in the pathogenesis of Crohn disease (CD) and ulcerative colitis (UC), the 2 major forms of inflammatory bowel disease (IBD), are not limited to those mediated by immune cells, but also include those mediated by nonimmune cells (1-6). An increasing number of reports have called attention to the significant contribution of epithelial, mesenchymal, nerve, and vascular cells as well as platelets to IBD pathogenesis $(3,7-10)$. It is now firmly established that the mucosal microvasculature is a nonimmune component crucially involved in intestinal inflammation (3).

Endothelial cells play a key role in mucosal immune homeostasis, acting as gatekeepers that regulate the type and number of leukocytes migrating from the intravascular to the interstitial space. Specialized microvascular cells, like those in high endothelial venules, selectively govern the influx of leukocytes into the gut (11). During intestinal inflammation, the mucosal microvasculature controls the nature and magnitude of such influx through chemokine secretion and expression of cell adhesion molecules (CAMs), which facilitate and amplify communication with leukocytes (12). The mucosal microvasculature is activated in IBD, as shown by aberrant

Nonstandard abbreviations used: aPC, activated PC; CAM, cell adhesion molecule; $\mathrm{CD}$, Crohn disease; ENA-78, epithelial-derived neutrophil-activating peptide 78; EPCR, endothelial PC receptor; HIMEC, human intestinal microvascular endothelial cell; IBD, inflammatory bowel disease; PC, protein C; TM, thrombomodulin; UC, ulcerative colitis.

Conflict of interest: B. Gerlitz and B.W. Grinnell are employees of Lilly Research Laboratories, a division of Eli Lilly \& Company Inc., which markets recombinant human activated protein C (Xigris) for treatment of high-risk severe sepsis.

Citation for this article: J. Clin. Invest. 117:1951-1960 (2007). doi:10.1172/JCI31027.
CAM expression and cytokine production as well as the presence of angiogenesis (13). A novel pathway crucially involved in controlling microvascular inflammation is the protein C (PC) pathway $(14$, 15). Although it was originally described as a major anticoagulant system $(16,17)$, it is now well established that the PC pathway is a crucial checkpoint for inflammation $(14,15,18)$.

Two important molecules in this pathway are thrombomodulin (TM) and endothelial PC receptor (EPCR), both of which are highly expressed on the surface of the microvasculature. An essential step for the pathway to become fully functional involves the capture of PC by the TM/EPCR complex on the endothelial surface and subsequent conversion to activated $\mathrm{PC}(\mathrm{aPC})$, a process that is catalyzed by thrombin $(16,17)$. This assembly process increases the rate of PC activation by more than 1,000-fold and at the same time blocks the ability of thrombin to activate platelets and the endothelium, an additional antiinflammatory step. A unique and critical feature of the PC pathway is its greater relevance to the microcirculation than to the macrocirculation. In fact, because the ratio of endothelial surface to blood volume is much higher in small rather than large vessels, there is a relative increase of TM concentrations in the microvasculature, promoting the conversion of $\mathrm{PC}$ to $\mathrm{aPC}$ at the local level $(14,16,17)$. Each component of the PC pathway, individually or in combination, has been shown to exert antiinflammatory activity and prevent microvascular inflammation $(14,15)$.

In particular, TM directly inhibits leukocyte adhesion to activated endothelium, and mice expressing a nonfunctional TM mutant show increased leukocyte influx in several models of inflammation (19). TM has also been shown to sequester the high-mobility group-B1 proteins, which are very potent proinflammatory molecules and thus prevent vascular activation (20). EPCR can block 

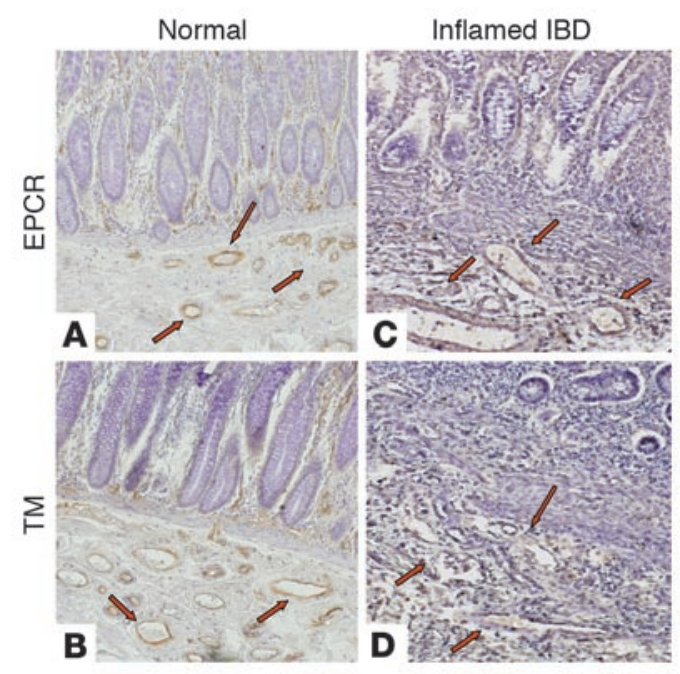

Noninflamed IBD

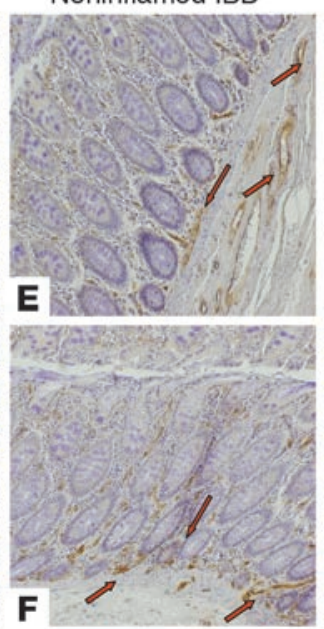

\section{Figure 1}

Immunohistochemical staining of EPCR and TM in colons of normal and actively inflamed IBD patients. The panels show brown immunohistochemical staining for EPCR and $\mathrm{TM}$ in the microvasculature of colonic mucosa and submucosa from histologically normal control (A and $\mathbf{B})$, active IBD (C and D), and noninflamed IBD ( $\mathbf{E}$ and $\mathbf{F})$ tissues. Images are representative of 12 control, $14 \mathrm{UC}$, and $15 \mathrm{CD}$ samples (original magnification, $\times 10$ ). Red arrows indicate intestinal microvessels. the integrin CD11b/CD18 on leukocytes and prevent leukocyte influx into tissues (21). After administration of LPS, mice with a severe deficiency of EPCR exhibit a reduced survival rate compared with wild-type mice, with a marked increase of neutrophilic tissue infiltration and enhanced production of chemokines (22). Finally, aPC inhibits the release of inflammatory cytokines such as TNF- $\alpha$, IL-6, and IL-8 in experimental models of endotoxin-induced inflammation $(23,24)$. aPC also limits leukocyte adhesion to the endothelium and extravasation into tissues $(25,26)$. In endothelial cell-based experimental systems, aPC inhibits a variety of inflammatory events in human umbilical vascular endothelial cells, such as CAM expression, cytokine production, and NF- $\mathrm{\kappa B}$ nuclear translocation $(27,28)$. Nevertheless, each of the antiinflammatory components of the PC pathway is downregulated in inflammation, as observed in various models of experimental inflammation. For instance, expression of TM on lung endothelial cells is suppressed by inflammation secondary to total-body irradiation (29). In the ovalbumin-induced bronchial asthma model, a decrease in aPC concentration was noted in the bronchoalveolar lavage associated with the downregulation of both TM and EPCR, as well as in atherosclerosis. (30). In animal models of LPS-induced sepsis, TM expression diminishes in a dose- and time-dependent manner in lung, liver, and renal peritubular capillaries (31).

Essentially no information exists on the expression and function of the PC pathway in human and experimental IBD, although it is well established that coagulation and inflammation are interdependent processes in IBD $(32,33)$. To date, no mechanistic study to our knowledge has been performed to investigate the regulatory mechanisms of TM and EPCR in IBD, the expression and function of the components of the PC pathway in the mucosal microvasculature, and their possible involvement in IBD pathogenesis. Therefore, the aim of the present study was to assess the expression and function of the PC pathway in IBD and to explore the therapeutic potential of manipulating such a pathway in experimental intestinal inflammation. In $C D$ and $U C$, the 2 major forms of IBD, there

Figure 2 was loss of expression of endothelial EPCR and TM, which in turns caused impairment of PC activation by the inflamed mucosal microvasculature. In isolated human intestinal endothelial cells, administration of recombinant activated PC had a potent antiinflammatory effect, as demonstrated by downregulated cytokine-dependent cell adhesion molecule expression and chemokine production as well as inhibited leukocyte adhesion. In vivo, administration of activated PC was therapeutically effective in ameliorating experimental colitis as evidenced by reduced weight loss, disease activity index, and histological colitis scores as well as inhibited leukocyte adhesion to the inflamed intestinal vessels. The results suggest that the PC pathway represents a new system crucially involved in governing intestinal homeostasis mediated by the mucosal microvasculature. Restoring the PC pathway may represent a new therapeutic approach to suppress intestinal inflammation in IBD.
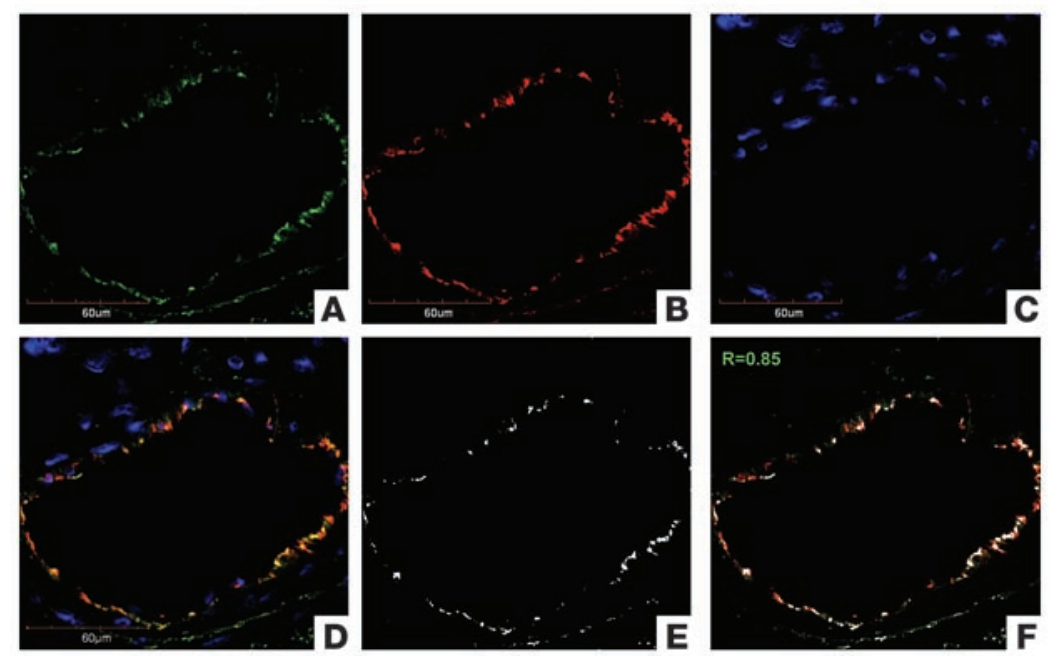

Localization of EPCR in IBD mucosa. Fluorescence micrographs of colonic mucosa microvessels in normal mucosa. (A-C) Staining for EPCR (green, A), von Willebrand factor (red, B), and nuclei (blue, C). (D) Merge (yellow) of panels A-C. (E and F) Binary mask of colocalization (white, $\mathbf{E}$ ) and colocalization of panels $\mathbf{B}$ and $\mathbf{E}(\mathbf{F})$. Images are representative of 6 control, $7 \mathrm{CD}$, and $8 \mathrm{UC}$ samples. Scale bars: $60 \mu \mathrm{m}$. 

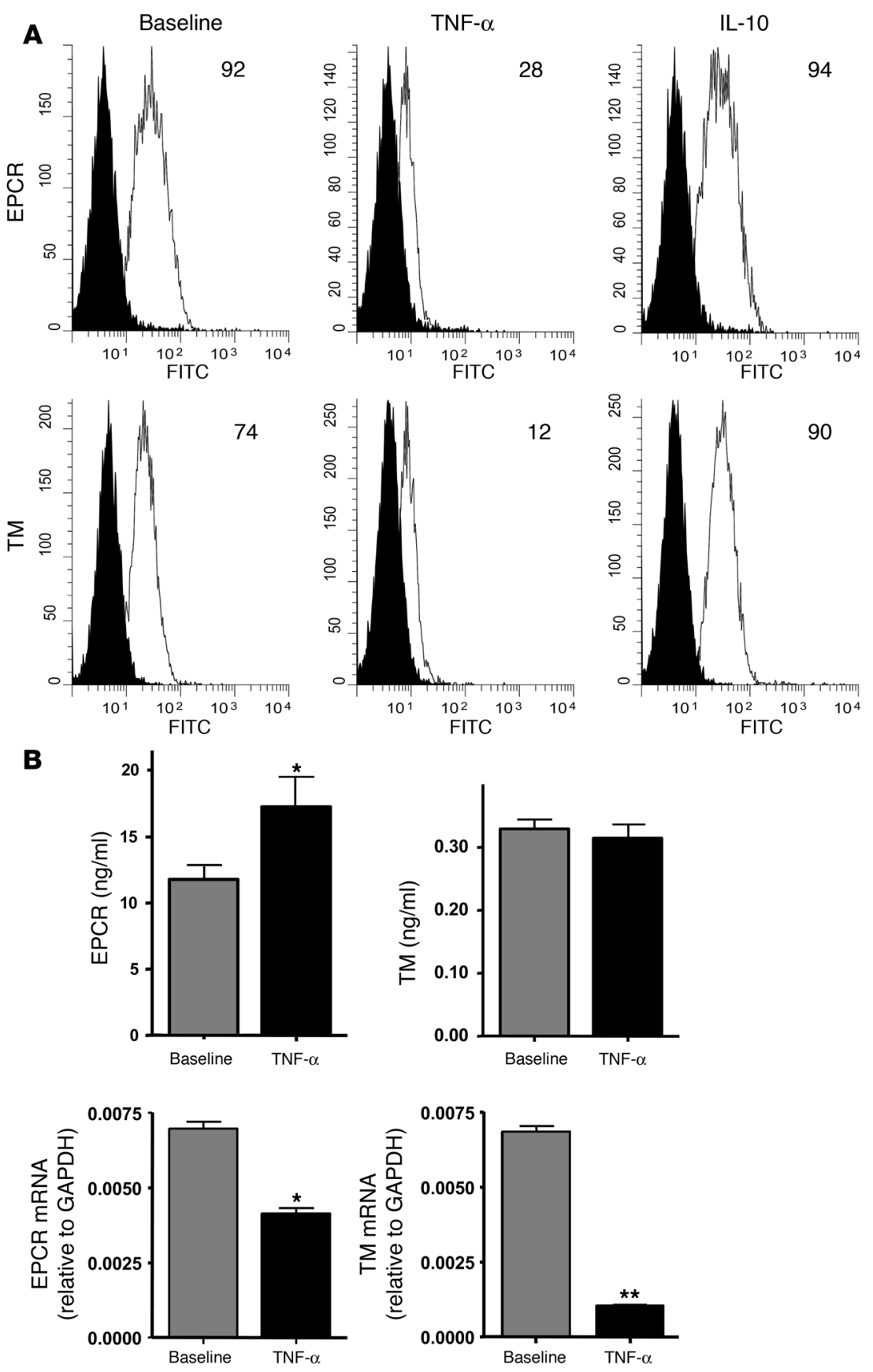

\section{Results}

Evidence for TM and EPCR downregulation in the IBD microvasculature. In order to investigate the expression levels of TM and EPCR in normal and IBD microvasculature, we initially performed immunohistochemical staining of 12 histologically normal control, 15 active CD-involved, and 14 UC-involved mucosa derived from human surgical specimens. Normal colonic mucosa showed strong vascular immunostaining for both EPCR and TM (Figure $1, \mathrm{~A}$ and $\mathrm{B})$. In contrast, microvessels of actively inflamed CD or UC mucosa expressed minimal levels of both proteins, without any apparent difference between the 2 forms of IBD (Figure 1, C and $\mathrm{D}$ ). To validate this visual assessment, semiquantitative scoring of tissue samples was performed, which confirmed significant

\section{Figure 3}

Effect of different cytokines on EPCR and TM expression by HIMECs. (A) HIMEC monolayers were left untreated (Baseline) or stimulated with TNF- $\alpha$ or IL-10 for 24 hours, after which cells were suspended and EPCR and TM expression measured by flow cytometry. Filled curve represents background signal from the isotype control. Values are representative of 6 separate experiments. Numbers represent the net percentage of positive cells. (B) HIMEC monolayers were left untreated or stimulated with TNF- $\alpha$ for 24 hours, after which supernatants were collected for ELISA measurement of soluble EPCR and TM. Cellular mRNA was extracted for real-time PCR of EPCR and TM message levels. Values are representative of 3 separate experiments. ${ }^{*} P<0.05$, ${ }^{\star \star} P<0.01$ versus baseline.

decreases of vascular EPCR expression in UC $(0.8 \pm 0.6$ normalized to GAPDH) and CD $(0.8 \pm 0.8$ normalized to GAPDH) compared with normal mucosa $(2.5 \pm 0.5$ normalized to GAPDH; $P<0.01$, both comparisons). Similar results were obtained for TM expression, which was significantly decreased in both UC $(0.6 \pm 0.7$ normalized to GAPDH) and $\mathrm{CD}$ $(0.9 \pm 0.7$ normalized to GAPDH $)$ vessels compared with those in normal control mucosa $(2.6 \pm 0.4$ normalized to GAPDH; $P<0.01$, both comparisons). No differences were found between control mucosa and noninflamed IBD, which showed high expression levels of both EPCR and TM (Figure 1, E and F).

To confirm the microvascular localization of the staining in the mucosa, we used fluorescence microscopy for detection of EPCR in conjunction with antibodies against von Willebrand factor (Figure 2, A and B). The specificity of EPCR endothelial cell origin was confirmed by the yellow staining of the merged images (Figure 2D) with the DAPI staining for nuclei (Figure 2C). Confocal microscopy was combined with quantitative analysis to confirm the extent of EPCR colocalization with von Willebrand factor $(r=0.85$; Figure 2, E and F). Additionally, TM colocalized with von Willebrand factor, demonstrating endothelial specificity (data not shown).

Effect of cytokines on TM and EPCR expression in human intestinal microvascular endothelial cells. The downregulation of TM and EPCR in involved IBD tissue suggests that the local proinflammatory microenvironment could inhibit their endothelial expression. To reproduce this event in vitro, we tested the effect of TNF- $\alpha$ and IL-1 $\beta, 2$ prototypical proinflammatory cytokines known to be elevated in IBD, on TM and EPCR expression in human intestinal microvascular endothelial cells (HIMECs). Essentially all unstimulated HIMECs expressed EPCR and TM on their surfaces, and stimulation by both TNF- $\alpha$ and IL- $1 \beta(50 \mathrm{ng} / \mathrm{ml}$ and $100 \mathrm{U} / \mathrm{ml}$, respectively; data not shown) dramatically downregulated these expression levels (Figure 3A). In addition to proinflammatory 


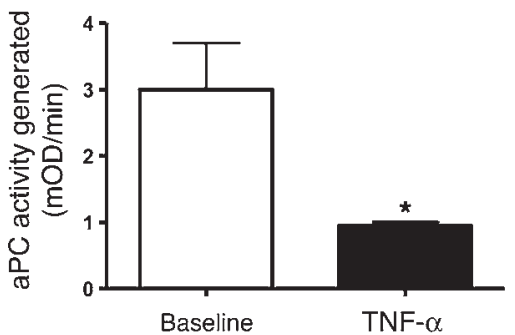

Figure 4

TNF- $\alpha$-inflamed HIMECs exhibit a reduced capacity for PC activation. HIMEC monolayers were incubated 24 hours with or without TNF- $\alpha$ and assayed for their ability to support activation of $P C$ by thrombin. Generation of aPC was measured using the chromogenic tripeptidylpNA substrate S-2366; relative capacity for PC activation is presented as change in absorbance at $405 \mathrm{~nm}$ over time ( $\mathrm{mOD} / \mathrm{min})$. Values are representative of 3 separate experiments. ${ }^{*} P<0.05$ versus baseline.

cytokines, we studied the potential effect of antiinflammatory molecules such as IL-10 $(5 \mathrm{ng} / \mathrm{ml})$ and TGF- $\beta$ on TM and EPCR expression. Notably, IL-10 had no effect on EPCR expression and modestly increased TM expression (Figure 3A). Comparable results were observed with TGF- $\beta$ (data not shown).

In order to assess whether the downregulatory effects of TNF- $\alpha$ on HIMECs are the result of shedding or of reduced transcription, we used the above experimental conditions to measure the concentration of soluble EPCR and soluble TM as well as cellular mRNA levels in HIMEC culture supernatants. TNF- $\alpha$ significantly increased soluble EPCR release $(P<0.05)$, but failed to stimulate TM shedding by HIMECs. Furthermore, TNF- $\alpha$ strongly suppressed both EPCR $(P<0.05)$ and TM $(P<0.01)$ at the transcriptional level (Figure 3B).

Inflammatory cytokines impair PC conversion into aPC. Because inflammation downregulates TM and EPCR levels, which in turn leads to impairment of PC conversion to aPC (16), we investigated whether HIMECs are able to generate aPC. We initially added exogenous human recombinant PC to unstimulated HIMECs and measured its conversion into $\mathrm{aPC}$ by assaying the generation of proteolytic activity toward a tripeptidyl chromogenic substrate. Under these resting conditions, aPC was readily detected in the HIMEC supernatants (Figure 4). To mimic the inflammatory state of the mucosal microvasculature in

\section{Figure 5}

Downregulation of VCAM-1 and ICAM-1 by recombinant aPC in TNF- $\alpha$-inflamed HIMECs. HIMEC monolayers were left untreated or stimulated with TNF- $\alpha$ in the presence or absence of recombinant human aPC. After 24 hours, cells were suspended and VCAM-1 and ICAM-1 expression was measured by flow cytometry. Filled curve represents background signal from the isotype control. Values are representative of 5 separate experiments. Numbers represent the net percentage of positive cells.
IBD, we stimulated HIMECs with TNF- $\alpha$ and measured PC activation. Under these inflammatory conditions, conversion of PC into aPC was drastically impaired (Figure 4), a response consistent with the concomitant downregulation of TM and EPCR (Figure 3A).

aPC is a potent antiinflammatory molecule. Having demonstrated that TNF- $\alpha$ stimulation impairs the generation of aPC and causes the loss of this antiinflammatory molecule, we next investigated whether supplementing HIMECs with aPC would eliminate or ameliorate microvascular inflammation. To test this possibility, HIMECs were either left untreated or stimulated with TNF- $\alpha$ in the absence or presence of $80 \mathrm{nM}$ human recombinant aPC. We investigated whether expression of VCAM-1 and ICAM-1, indicators of the antiinflammatory function of aPC, was inhibited. As previously reported (34), VCAM-1 and ICAM-1 were readily upregulated by TNF- $\alpha$ as detected by flow cytometry (Figure 5). Notably, incubation of HIMECs with aPC both prevented the upregulation of CAMs (data not shown) and inhibited their TNF- $\alpha$-induced upregulation (Figure 5). Similar results were obtained when IL-1 $\beta$ was used as the inflammatory stimulus (data not shown).

In addition to upregulating CAM, inflamed endothelial cells secrete a large variety of chemokines. Using the same experimental system reported above, we measured the concentration of 3 major chemokines implicated in inflammatory cell recruitment in IBD: epithelial-derived neutrophil-activating peptide 78 (ENA-78), IL-8, and MCP-1. HIMECs produced higher levels of chemokines upon TNF- $\alpha$ stimulation, an effect that was significantly inhibited by the addition of aPC to the cultures, although levels did not return to baseline (Figure 6). Stimulation with IL-1 $\beta$ replicated these results (data not shown).

aPC inhibits $T$ cell adhesion to inflamed endothelium. It is well known that under inflammatory conditions, endothelium-leukocyte adhesion markedly increases. This aberrant $\mathrm{T}$ cell interaction is a key pathogenic event in IBD, and its blockade is currently being tested as a novel therapeutic strategy in both CD and UC (35, 36). Based on our demonstration that aPC inhibited both CAM and chemokine production, it is to be expected that aPC would also inhibit $\mathrm{T}$ cell adhesion. Hence, using the same experimental system described above, we quantified the adhesion of $\mathrm{T}$ cells to inflamed HIMECs in the presence or absence of exogenous aPC.
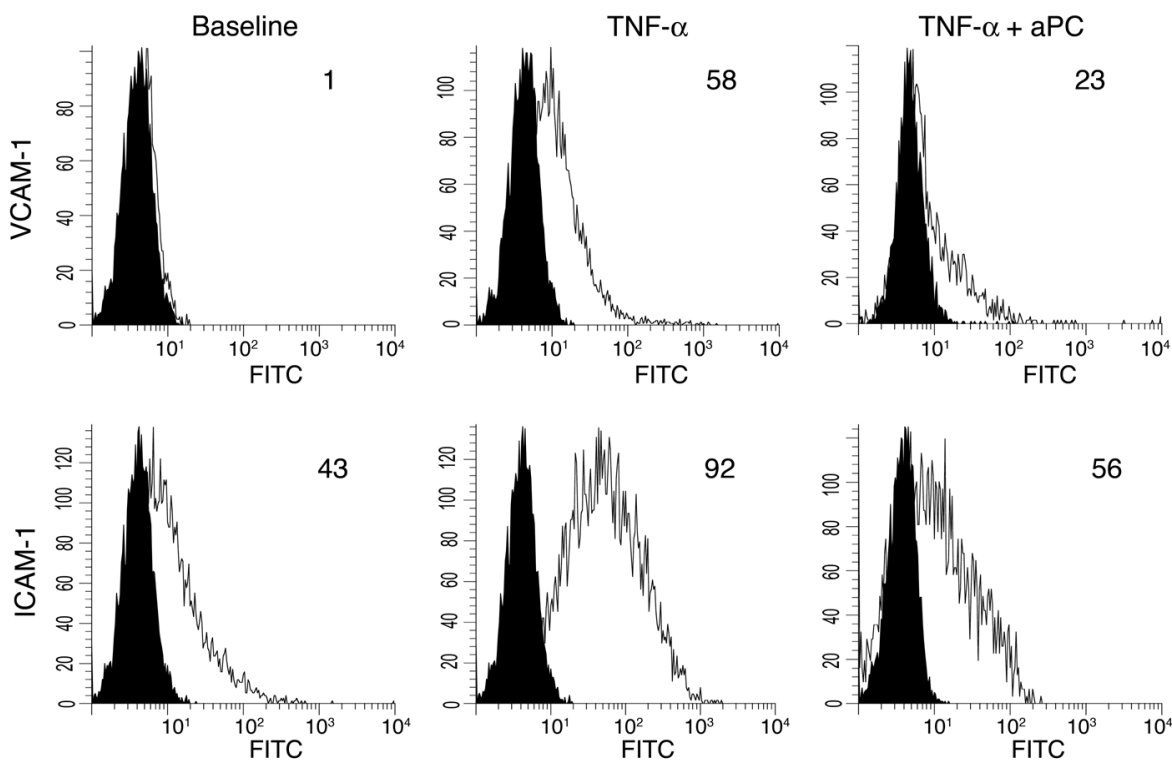

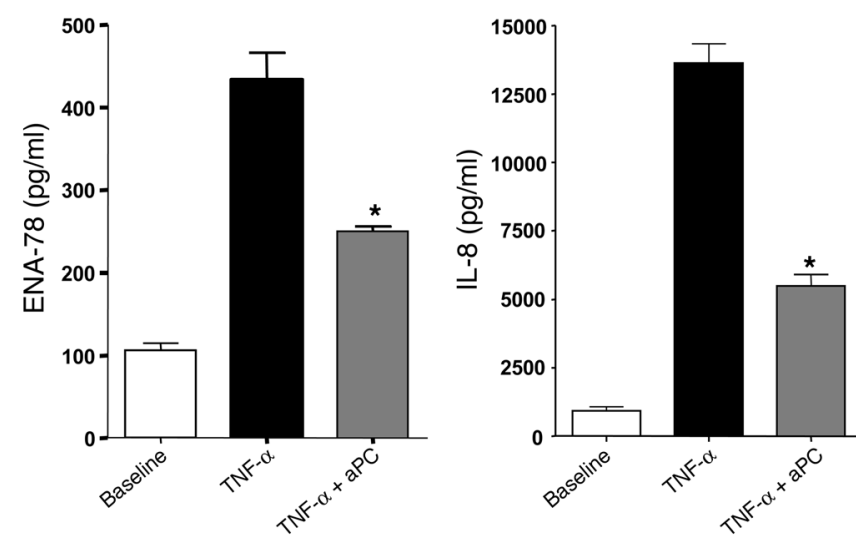

Unstimulated HIMECs bound few T cells, but adhesion markedly increased after TNF- $\alpha$ stimulation (Figure 7). More importantly, the addition of aPC significantly inhibited $\mathrm{T}$ cell adhesion to inflamed HIMECs $(P<0.05$; Figure 7$)$.

Impairment of $P C$ activation in dextran sodium sulfate-induced murine colitis. Inflammation downregulates the expression of TM and EPCR in several experimental models of inflammation, resulting in an impairment of PC activation $(16,17)$. As experimental colitis is characterized by extensive intestinal microvascular inflammation $(37,38)$, this should be followed by a decrease of PC conversion into aPC in the inflamed mucosa. To verify this, we injected healthy mice and those with dextran sodium sulfate-induced (DSS-induced) colitis with recombinant $\mathrm{PC}$ and measured the in vivo activation of PC by a colorimetric reaction, using a standard curve with known quantities of aPC in pooled murine plasma (19). A marked and significant decrease of PC conversion into aPC was observed in colitic mice versus healthy controls $(P<0.05$; Figure 8$)$.

Effect of aPC administration on DSS-induced murine colitis. Based on the above results, we next investigated whether restoring normal levels of aPC would be beneficial in experimental colitis by assessing the potential therapeutic effect of aPC administration in the DSS colitis model. Mice received either 0.1 or $1 \mathrm{mg} / \mathrm{kg}$ murine recombinant $\mathrm{aPC}$ or its vehicle, and the colitis disease activity index (DAI) (39-41) and colonic histology were assessed over a period of 10 days. Compared with vehicle-treated control animals, aPC-treated mice displayed less severe colitis, as demonstrated by less severe weight loss $(P<0.05$; Figure $9 \mathrm{~A})$ and an improved DAI $(P<0.05$; Figure 9B). In addition, at the end of the 10-day treatment schedule, histological assessment showed that aPC-treated mice displayed a marked decrease of inflammation compared with control animals (Figure 9, C and D). No significant differences were found between the 0.1 and $1 \mathrm{mg} / \mathrm{kg}$ doses (data not shown). Notably, despite the fact that aPC is a natural anticoagulant, administration of aPC failed to induce any bleeding events in the treated animals.

Effect of aPC administration on in vivo leukocyte adhesion in the intestinal microcirculation. Having shown that recombinant aPC inhibits leukocyte adhesion to HIMECs in vitro and that aPC administration improves experimental colitis, we next investigated whether the therapeutic effect of recombinant $\mathrm{aPC}$ was mediated by the inhibition of CAM and chemokine production in the intestine followed by inhibition of leukocyte adhesion to the microvascular endothelium in vivo. Mice treated with aPC displayed reduced intestinal levels of VCAM-1, ICAM-1, and IL-8 compared with pla-

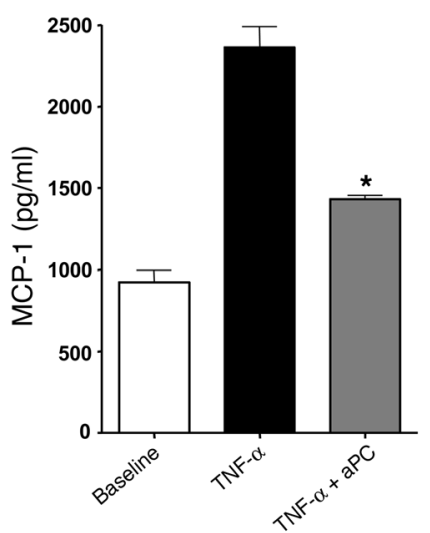

Figure 6

Downregulation of chemokine production by recombinant aPC in TNF- $\alpha$-inflamed HIMECs. HIMEC monolayers were left untreated or stimulated with TNF- $\alpha$ in the presence or absence of recombinant human aPC. After 24 hours, supernatants were collected, and ENA-78, IL-8, and MCP-1 were measured by ELISA. Values are representative of 5 separate experiments. ${ }^{*} P<0.05$ versus TNF- $\alpha$ without aPC. cebo-treated mice (Figure 10, A and B). In order to assess whether such inhibition was functional, we quantified the number of adhering leukocytes in the colonic microcirculation by intravital microscopy (42). In healthy mice, very few leukocytes adhered to colonic venules (Figure 10C). In contrast, a large number of leukocytes adhered to the colonic microvascular endothelium in mice with DSS-induced colitis $(P<0.001)$. This difference was dependent on a loss of aPC in the microcirculation of the inflamed colonic mucosa, as demonstrated by treatment of DSS colitic mice with recombinant murine $\mathrm{aPC}$, which reduced the number of adhering leukocyte to the colonic venules by $40 \%$ compared with untreated DSS colitic mice $(P<0.05$; Figure 10$)$.

\section{Discussion}

The results of the present study show that the PC pathway was defective in both CD and UC patients and that this system was crucial for governing microvascular inflammation in the gut. More importantly, restoring the function of the PC pathway may be a new therapeutic approach for translational studies in IBD.

Under physiological circumstances, the tissue microcirculation functions in an anticoagulant and antiinflammatory fash-

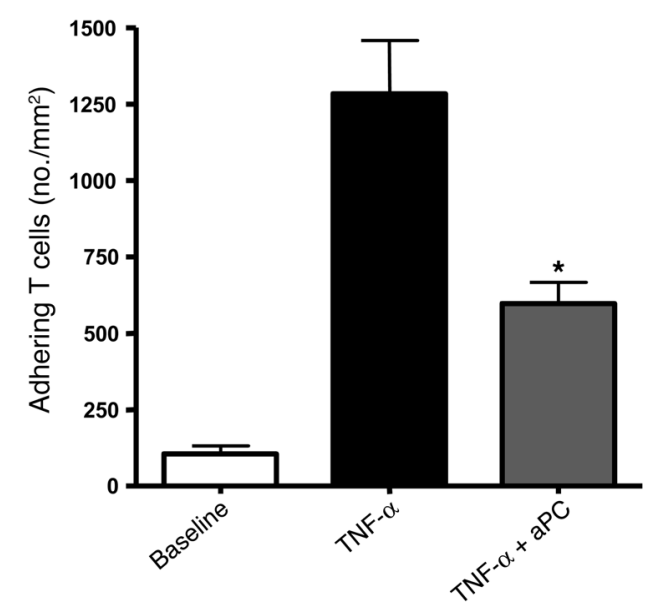

\section{Figure 7}

Inhibition of T cell adhesion to TNF- $\alpha$-inflamed HIMECs by recombinant aPC. HIMEC monolayers were left untreated or stimulated with TNF- $\alpha$ with or without recombinant human aPC. Calcein-labeled MOLT4 T cells were added to the HIMEC monolayers. Values are mean \pm SEM of 4 separate experiments. ${ }^{*} P<0.05$ versus TNF- $\alpha$ without aPC. 


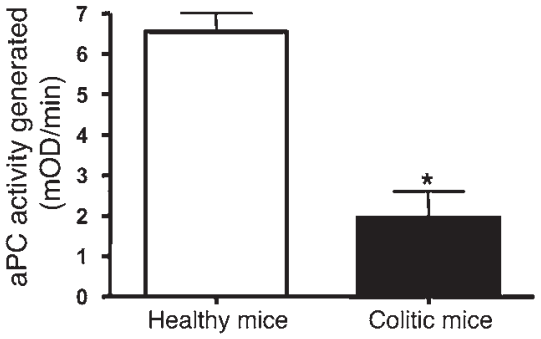

ion, both actions being finely tuned by the PC pathway $(15,16$, 43-46). When microvascular inflammation occurs, a number of processes are set in motion, including coagulation, angiogenesis, and immune responses (46-48). The coagulation pathway is one of the first to be elicited, and its activation is followed by proinflammatory phenomena, in part because of the loss of the antiinflammatory activity of the PC pathway. In several inflammatory conditions such as lung inflammation, atherosclerosis, and sepsis there is marked downregulation of both TM and EPCR, leading to a functional impairment of PC conversion into aPC $(16,17)$. Because mucosal microvascular inflammation and coagulation/ thrombosis are well-documented events in IBD (32), it would be reasonable to hypothesize that TM and EPCR would be downregulated (3). Indeed, in vivo we found reduced or absent expression of both components of the PC pathway in the microvasculature of CD and UC compared with normal mucosa. Such downregulation appeared to be present in both forms of IBD and occurred in

\section{Figure 8}

Dextran sodium sulfate (DSS) colitic mice exhibit a reduced capacity for PC activation. Healthy and colitic mice ( $n=3$ per group) were injected i.v. with exogenous human PC. After 30 minutes, blood was collected into sodium citrate anticoagulant supplemented with benzamidine to protect aPC from inhibition by endogenous irreversible inhibitors. Using the immunocapture assay described in Methods, aPC was measured using the chromogenic tripeptidyl-pNA substrate S-2366; relative capacity for $\mathrm{PC}$ activation is presented as the change in absorbance at $405 \mathrm{~nm}$ over time. ${ }^{*} P<0.05$ versus healthy mice. areas of active mucosal inflammation. To complement the results of these descriptive but essential data with a more mechanistic approach, we reproduced in vitro the downregulation of TM and EPCR by stimulating HIMECs with proinflammatory cytokines in order to mimic the IBD inflamed milieu. Both TNF- $\alpha$ and IL-1 $\beta$ triggered prompt surface downregulation of TM and EPCR, an event caused by inhibition of RNA transcription for TM and by both increased shedding of the soluble form of EPCR and inhibition of RNA transcription for EPCR, thus highlighting the crucial role of inflammatory mediators in regulating expression of TM and EPCR. Furthermore, to study the functional consequence of TM and EPCR downregulation in HIMECs, we studied the cells' capacity to activate $\mathrm{PC}$ in resting and inflammatory conditions. As expected, HIMECs activated PC in resting conditions, but this process was strongly inhibited after treatment with proinflammatory cytokines, consistent with the downregulation of TM and EPCR and reinforcing the notion that inflammation impairs PC activa-
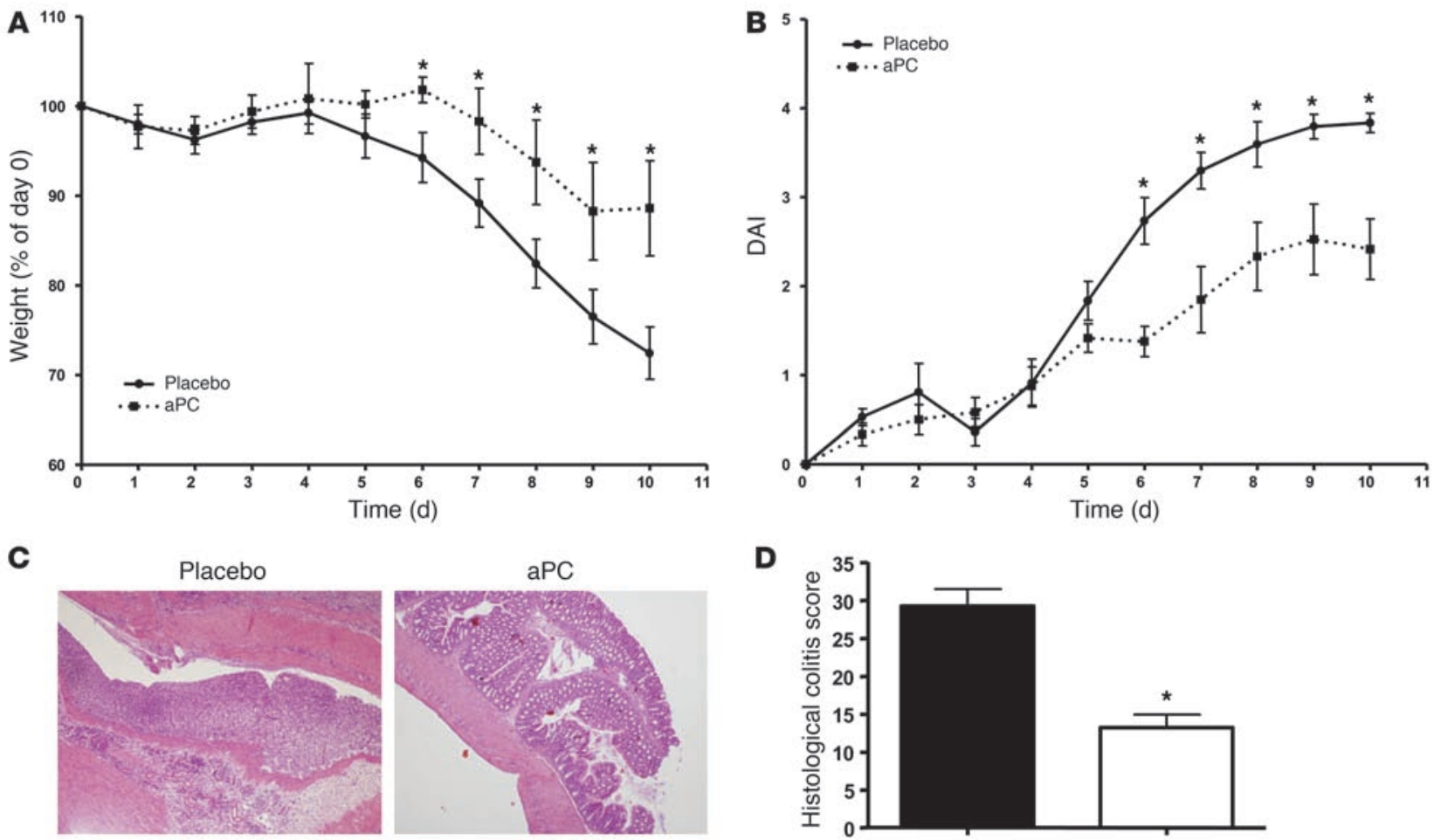

D

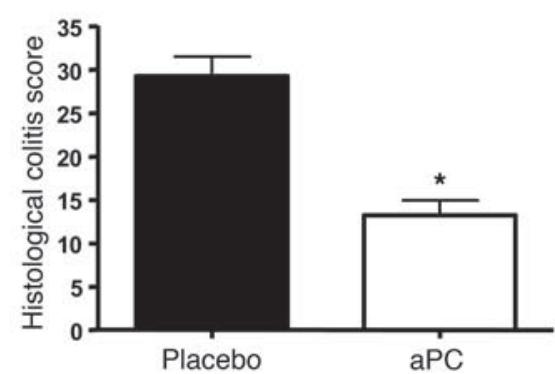

Figure 9

Therapeutic effects of murine recombinant aPC administration. Animals undergoing DSS treatment were given daily i.v. injections of recombinant murine aPC (1 mg/kg; $n=10)$ or placebo $(n=12)$. Weight was monitored $(\mathbf{A})$ as well as disease activity index (DAl) (B). After 10 days, mice were sacrificed, and colons were assessed for histological colitis (C and $\mathbf{D}) .{ }^{*} P<0.05$, aPC versus placebo. 

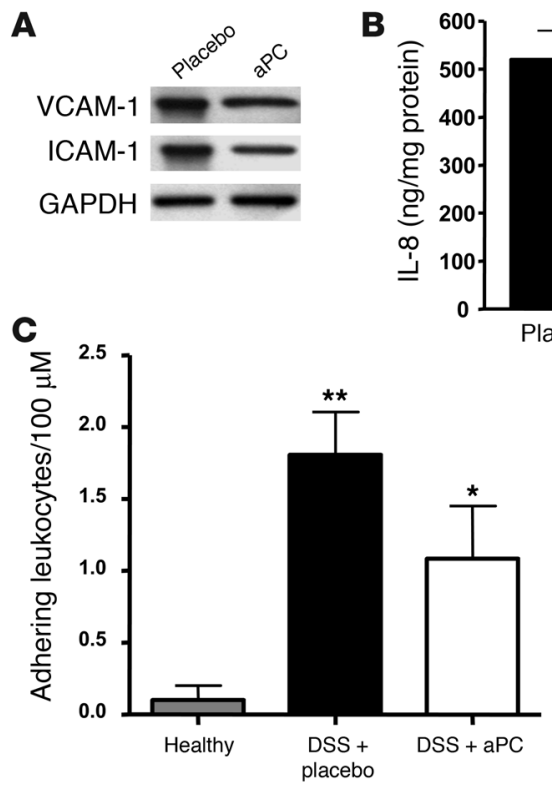

tion. Taken together, our in vivo and in vitro data suggest that in IBD mucosal microcirculation, expression and function of the PC pathway are impaired.

In order to investigate whether the observed impaired PC pathway activity could have some therapeutic implication, we supplemented HIMECs with exogenous recombinant aPC and assessed its antiinflammatory effects by studying CAM expression, chemokine production, and leukocyte adhesion to endothelium. In vitro, aPC was a potent antiinflammatory agent: it inhibited TNF- $\alpha$-induced CAM upregulation and secretion of chemokines including MCP-1, which was already produced at high levels by unstimulated HIMECs. Because CAM upregulation and chemokine production by HIMECs are crucial for leukocyte recruitment into inflamed intestine, we assessed whether the inhibition induced by aPC was functional. We performed a series of T cell-HIMEC adhesion assays and consistently observed that aPC exerted its antiinflammatory activities ultimately by inhibiting $\mathrm{T}$ cell adhesion.

Based on our results using human cells, we also hypothesized that during the course of experimental colitis, there would be a progressive impairment of the rate of PC conversion. In agreement with our human data, we found that colitic mice exhibited an impaired capacity to convert $\mathrm{PC}$ into aPC, thus reinforcing the concept that during gut inflammation the PC pathway is downregulated and cannot exert its physiological antiinflammatory tasks in the microcirculation.

Based on our understanding of the expression and function of PCs in various inflammatory processes, the important therapeutic implications of manipulating the PC system have begun to emerge (49). In several experimental models in which the conversion of PC into aPC is impaired, including asthma, sepsis, and ischemia/reperfusion injury, administration of recombinant aPC has consistently proved to be effective in inhibition of cytokine production, CAM expression, and leukocyte influx into the tissues $(26,30,50-57)$. Therefore, we tested the therapeutic efficacy of murine recombinant aPC administration in restoring the functionality of the PC pathway. Importantly, a beneficial therapeutic effect was observed in the DSS model of colitis, resulting in significant reduction in DAI, decreased weight loss, and improvement

\section{Figure 10}

aPC inhibits CAM expression, IL-8 production, and leukocyte adhesion to the endothelium in the intestine. Animals undergoing DSS treatment were given daily i.v. injections of recombinant murine aPC or placebo. After 10 days mice were sacrificed, and biopsies were collected for Western blot analysis for VCAM-1 and ICAM-1 (A) or organ culture for IL-8 measurement by ELISA (B). (C) Leukocyte-endothelial cell interactions were assessed by intravital microscopy in 3 groups of animals: healthy mice $(n=5)$; DSS colitic mice treated for 10 days with placebo $(n=8)$; and DSS colitic mice treated for 10 days with daily i.v. injections of recombinant aPC $(n=6)$. For each animal, 3-6 unbranched venules were studied and the mean value was calculated. Leukocyte adhesion is expressed as the number of firmly adherent leukocytes to the endothelium per $100 \mu \mathrm{m}$ of venule. ${ }^{*} P<0.05$ versus placebo; ${ }^{* *} P<0.001$ versus healthy mice.

of the colitis histological score. Our results provide evidence that restoring the PC pathway could be beneficial for IBD treatment. Because our in vitro studies showed that aPC inhibited leukocyte adhesion to endothelium, and because blocking leukocyteendothelial interactions is a very promising approach for IBD, we used intravital microscopy to investigate whether administration of aPC in colitic mice would affect leukocyte rolling and adhesion to intestinal endothelium. Our results showed that aPC administration inhibited leukocyte adhesion to the inflamed intestinal microcirculation using intravital microscopy, thus supporting its antiinflammatory effects in experimental colitis. Taken together, our observations represent a proof-of-concept for the biological importance of and the antiinflammatory role played by the PC pathway in intestinal inflammation. However, future studies using genetically manipulated mice such as $\mathrm{PC}^{-/-}, \mathrm{EPCR}^{\delta / \delta}$, and $\mathrm{TM}^{\mathrm{pro} / \mathrm{pro}}$ mice will help to address the mechanism whereby defects in PC are associated with amplification of inflammation and whether our therapeutic effects by using recombinant aPC are dependent or independent of EPCR or TM.

The complexity of IBD is well recognized, stemming from limited knowledge about its cause and mechanisms and uncertainty as to whether original pathogenic events persist throughout its course or whether secondary phenomena take over control of inflammation. Until these issues are resolved, it is appropriate to focus on all components whose manipulation may disrupt the cycle of chronic gut inflammation. Given the relevance of immune-nonimmune interactions to IBD, we propose that the endothelial PC pathway is a crucial system in governing microvascular inflammation at the intestinal mucosal level. Our studies complement and strengthen the mounting body of evidence pointing to vascular inflammation as a key pathogenic component of autoimmune and chronic inflammatory disorders. Considering that the treatment of IBD is still a major practical challenge and that the heterogeneity of the patient response to therapy is likely a result of the heterogeneity of mechanisms of inflammation, the significance of our study is further reinforced by the possibility that manipulation of the PC pathway can offer another tool in the therapeutic arsenal against IBD and may be a justifiable new focus for translational studies in both CD and UC.

\section{Methods}

Immunostaining and confocal imaging. Immunohistochemical staining for TM and EPCR was performed as previously reported (58). Intestinal tissues were obtained from surgical specimens of patients with CD, UC, and as control, 
from normal areas of the intestine of patients admitted for bowel resection because of colon cancer, polyps, or diverticulosis. Specimens were fixed in $10 \%$ formalin. Written informed consent was obtained from all patients. Parafin-embedded intestinal sections were cut at $3 \mu \mathrm{m}$ thickness, deparaffinized, hydrated, blocked for endogenous peroxidase using $3 \% \mathrm{H}_{2} \mathrm{O}_{2} / \mathrm{H}_{2} \mathrm{O}$, and subsequently subjected to microwave epitope enhancement using a Dako Target retrieval solution (Dako). Incubation with the primary antibodies for TM (Santa Cruz Biotechnology Inc.) and EPCR (kindly provided by C.T. Esmon, Oklahoma Medical Research Foundation, Oklahoma City, Oklahoma, USA) was performed at 1:200 dilution for 30 minutes at room temperature. Detection was achieved using a standard streptavidin-biotin system (Vector Laboratories), and antigen localization was visualized with 3'-3-diamino benzidene (Vector Laboratories). Quantification of TM and EPCR expression was performed on immunostained sections by a semiquantitative method (scores from 0 to 3 ) as previously described (39).

Double fluorescence staining, microscopy, and imaging were performed as previously reported (13) using an IX51 Olympus Fluorescence microscope equipped with 3 lasers and photodetectors that permit detection of 3 separate fluorochromes.

Paraffin sections from histologically normal control and IBD-involved and uninvolved colonic mucosa were fixed, deparaffinized, blocked in HBSS containing $2 \%$ FBS for 30 minutes at $25^{\circ} \mathrm{C}$, and stained with antibodies to TM (Santa Cruz Biotechnology Inc.), EPCR (kindly provided by C.T. Esmon), or von Willebrand factor (diluted 1:100; Dako), all in HBSS with $2 \% \mathrm{FBS}$ overnight at $4^{\circ} \mathrm{C}$. For fluorescence staining, anti-rabbit IgG FITC conjugate (diluted 1:1,000; Sigma-Aldrich) and anti-mouse IgG R-phycoerythrin conjugate $\mathrm{F}(\mathrm{ab})_{2}$ (diluted 1:500; Sigma-Aldrich) were used as secondary antibodies, both diluted in a $1 \%$ FCS PBS solution and incubated with coverslips for 1 hour at $25^{\circ} \mathrm{C}$. All slides were rinsed 3 additional times and blotted, and coverslips were placed using VECTASHIELD Mounting Medium containing DAPI (Vector Laboratories). The slides were sealed and stored at $-20^{\circ} \mathrm{C}$ until examined by fluorescence microscopy. Differential interference contrast (Nomarski technique) was also used. Overlay images were assembled, and BP-IMARIS-4.2 Imaris Colocalization 4.2 software (Bitplane AG) was used for quantitative colocalization and statistical analysis. Human studies were approved by Istituto Clinico Humanitas and Catholic University of Rome.

Flow cytometric studies for detection of cell surface molecules and ELISA. Surgical specimens used for isolation of HIMECs were all of colonic origin. Written informed consent was obtained from all patients. Mucosal strips were obtained from surgically resected tissue and processed as previously described (34). Briefly, processing consisted of enzymatic digestion followed by gentle compression to extrude endothelial cell clumps, which adhered to fibronectin-coated plates and were subsequently cultured in MCDB131 medium supplemented with 20\% FBS, antibiotics, heparin, and endothelial cell growth factor. Cultures of HIMECs were maintained at $37^{\circ} \mathrm{C}$ in $5 \% \mathrm{CO}_{2}$, fed twice weekly, and split at confluence. HIMECs were used between passages 3 and 10 .

In order to determine TM, EPCR, VCAM-1, and ICAM- 1 expression by HIMECs, endothelial cells were plated on fibronectin-coated wells of a 24-well cluster plate at a density of $5 \times 10^{4} \mathrm{cells} / \mathrm{ml}$ in each well. Confluent monolayers were cultured for 24 hours in the presence of $50 \mathrm{ng} / \mathrm{ml} \mathrm{TNF}-\alpha$, $100 \mathrm{U} / \mathrm{ml} \mathrm{IL}-1 \beta, 5 \mathrm{ng} / \mathrm{ml} \mathrm{IL-10}$, and $5 \mathrm{ng} / \mathrm{ml} \mathrm{TGF}-\beta$ (R\&D Systems) with or without recombinant human aPC prepared as previously described (59). At the end of the culture, HIMECs were washed 5 times in cold PBS, and a single-cell suspension was obtained using a detaching buffer (PBS; $20 \mathrm{mM}$ HEPES, pH 7.4; 10 mM EDTA; and 0.5\% BSA), followed by vigorous pipetting. After additional washing, HIMECs were incubated with primary antiTM or anti-EPCR; washed; incubated with secondary mouse-FITC antibody, FITC-conjugated anti-VCAM-1 or anti-ICAM-1 antibody, or the appropri- ate isotype control antibody (BD Biosciences - Pharmingen) for 30 minutes at $4{ }^{\circ} \mathrm{C}$; fixed in $1 \%$ paraformaldehyde; and analyzed by flow cytometry as previously described (34). The above reported HIMEC stimulations did not alter cell viability, as assessed by trypan blue staining (data not shown).

Samples were analyzed by quantitative flow cytometry using a Coulter Epics XL Flow Cytometer (Beckman Coulter), and each analysis was performed on at least 10,000 events. Quantification of surface molecule expression was obtained using WinList software (version 5.0; Verity Software House).

Using the same conditions reported above for HIMEC stimulation, cell supernatants were harvested by centrifugation at $800 \mathrm{~g}$ for 5 minutes at $4^{\circ} \mathrm{C}$ and stored at $-70^{\circ} \mathrm{C}$. Supernatant samples were thawed once and analyzed for IL-8, MCP-1, and ENA-78 content in duplicate using a commercially available ELISA kit with an assay reproducibility greater than 95\% (R\&D Systems) or for soluble EPCR and soluble TM (Diagnostica Stago Inc.).

Real-time PCR. TM and EPCR gene expression was assessed in unstimulated and TNF- $\alpha$-activated (50 ng/ml) HIMECs. The cells were washed with cold PBS twice, and total RNA was isolated by TRIzOL Reagent (Invitrogen) and quantified by OD (see Supplemental Methods; available online with this article; doi:10.1172/JCI31027DS1).

Measurement of in vitro and in vivo capacity for $P C$ activation. HIMEC monolayers were incubated for an additional 24 hours in the presence or absence of $50 \mathrm{ng} / \mathrm{ml} \mathrm{TNF-} \alpha$ (R\&D Systems). The cells' capacity for supporting PC activation was then measured essentially as previously described (ref. 60 and Supplemental Methods). For the measurement of in vivo PC activation potential, $100 \mu \mathrm{g}$ human PC (Sigma-Aldrich) was injected i.v. into colitic and healthy C57BL/6 mice. After 20 minutes, mice were sacrificed, and blood was immediately collected into $3.8 \%$ sodium citrate anticoagulant supplemented with $500 \mathrm{mM}$ benzamidine- $\mathrm{HCl}$ ( 9 parts blood, 1 part sodium citrate/benzamidine). The small, "reversible" active site inhibitor benzamidine served to halt the irreversible inhibition of aPC by endogenous serine protease inhibitors (serpins) found in blood. Plasma was then prepared, and human aPC amidolytic activity was measured using an enzyme capture assay essentially as described previously (61). In brief, mouse plasma samples as described above were diluted further in benzamidine-containing buffer and then loaded into 96-well plates that had been coated with an anti-human PC antibody. Following incubation to immunocapture the aPC, plates were washed to remove all protease inhibitors (mouse serpins and benzamidine), the chromogenic substrate S-2366 was added to a concentration of $500 \mu \mathrm{M}$, and the aPC amidolytic activity at room temperature was monitored in a plate reader as the change in absorbance at $405 \mathrm{~nm}$ over time. Under these conditions, less than $15 \%$ of initial PC substrate was activated.

HIMEC-T cell adhesion assay. Adhesion assays were performed as previously reported $(34,58)$. The MOLT-4 T cell line (ATCC no. CRL-1582), kept under exponential growth conditions, was used for the adhesion assay. Confluent HIMEC monolayers were left untreated or stimulated with $50 \mathrm{ng} / \mathrm{ml} \mathrm{TNF-} \alpha$. After 1 hour, $10^{6}$ MOLT- 4 cells labeled with calcein (Invitrogen) were overlaid and allowed to adhere at $37^{\circ} \mathrm{C}$ in a $5 \% \mathrm{CO}_{2}$ incubator. Following 1 hour of coculture, nonadherent MOLT- 4 cells were removed by 3 sequential rinsings and washing with cold PBS. Fluorescent adherent cells were quantified in 10 randomly selected fields by an imaging system (Image-Pro Plus; Media Cybernetics) connected to an Optronics Color digital camera (Olympus) on an inverted fluorescence microscope. All experiments were performed in duplicate wells, and results are expressed as adherent cells per square millimeter.

Animal studies. Animal studies were approved by the Ethical Committee of Catholic University of Rome and Hospital Clinic y Provincial, Barcelona, Spain. C57BL/6 mice were used for the colitis model by administration of $2.5 \%$ DSS (molecular mass, $40 \mathrm{kDa}$; MP Biomedicals) in filter-purified 
(Millipore) drinking water for 10 days. Clinical DAI ranging from 0 to 4 was calculated using the following parameters: stool consistency (normal, loose, diarrhea), presence or absence of fecal blood (guaiac paper test and macroscopic evaluation of the anus), and weight loss (39-41).

For each animal, histological examination was performed on 3 samples of the distal colon; samples were fixed in $10 \%$ formalin before staining with $\mathrm{H} \& \mathrm{E}$. All histological quantitation was performed blinded using a scoring system previously described (40). The 3 independent parameters measured were severity of inflammation (0-3: none, slight, moderate, severe), extent of injury (0-3: none, mucosal, mucosal and submucosal, transmural), and crypt damage (0-4: none, basal one-third damaged, basal two-thirds damaged, only surface epithelium intact, entire crypt and epithelium lost). The score of each parameter was multiplied by a factor reflecting the percentage of tissue involvement $(\times 1,0 \%-25 \% ; \times 2,26 \%-50 \% ; \times 3,51 \%-75 \% ; \times 4,76 \%-100 \%)$ and all numbers were summed. Maximum possible score was 40 .

Treatment with recombinant murine aPC $(0.1$ or $1 \mathrm{mg} / \mathrm{kg})$, prepared as previously described (62), or its vehicle was done by i.v. injections daily for 10 days. The drug was brought to room temperature before injection and delivered in a volume of $0.2 \mathrm{ml}$ per injection per mouse.

At the end of the treatment, mice were sacrificed, and mucosal samples were collected for protein extraction and Western blot analysis for VCAM-1 and ICAM-1 (Santa Cruz Biotechnology Inc.) as previously reported (13). Murine colonic biopsies were collected and maintained in organ culture as previously reported (63). After 24 hours, supernatants were collected, centrifuged at $900 \mathrm{~g}$ for 15 minutes, assayed for protein concentration (BioRad), and frozen until ELISA for IL-8 (murine homolog KC) was preformed (R\&D Systems), as previously reported (13).

Intravital microscopy study of lenkocyte-endothelium interactions in the bowel microvasculature. Mice were anesthetized with s.c. ketamine $(150 \mathrm{mg} / \mathrm{kg})$ and xylazine $(7.5 \mathrm{mg} / \mathrm{kg})$, and a tail vein was cannulated. Throughout the experiments, rectal temperature was monitored using an electrothermometer and was maintained between $36.5^{\circ} \mathrm{C}$ and $37.5^{\circ} \mathrm{C}$ with an infrared heat lamp. The abdomen was opened via a midline incision, and a segment of the distal colon was chosen for microscopy examination, exteriorized, and covered with a cotton gauze soaked with bicarbonate buffer, as previously described (42). Mice were then placed on an adjustable microscope stage, and the colon was extended over a nonautofluorescent coverslip that allowed observation of a $2-\mathrm{cm}^{2}$ segment of tissue. An inverted microscope (Diaphot 300; Nikon) with a CF Fluor 403 objective lens (Nikon) was used. A charge-coupled device (CCD) camera (model XC-77; Hamamatsu Photonics) with a C2400 CCD camera control unit and a C2400-68 intensifier head (Hamamatsu Photonics), which was mounted on the microscope, projected the image onto a monitor (Trinitron KX-14CP1; Sony), and the images were recorded using a videocassette recorder (SR-S368E; JVC) for offline analysis. Leukocytes were in vivo labeled by s.c. injection of rhodamine-6G (Invitrogen). Rhodamine-6G-associated fluorescence was visualized by epi-illumination at $510-560 \mathrm{~nm}$, using a $590-\mathrm{nm}$ emission filter. Single unbranched submucosal and lamina propria venules with internal diameters of 25-40 mm were selected for observation. The flux of rolling leukocytes, leukocyte rolling velocity, number of adherent leukocytes, venular blood flow, and venular wall shear rate were determined offline after playback of the videotapes, as previously described (42). Rolling leukocytes were defined as those white blood cells that moved at a velocity less than that of free-flowing leukocytes in the same vessel. The flux of rolling leukocytes was measured as the number of rolling leukocytes that passed a fixed point within a small $(10 \mathrm{~mm})$ viewing area of the vessel during a 1-minute period. Leukocytes were considered adherent to venular endothelium when stationary for 30 seconds or longer, and adherent leukocyte values were expressed as the number per 100-mm length of venule. In each animal, 3-6 random venules were examined, and results were calculated as the mean of each parameter in all venules examined.

Statistics. Data were analyzed by GraphPad 4.0 software and expressed as mean \pm SEM. Student's $t$ test or ANOVA followed by the appropriate post-hoc test were used as appropriate. A $P$ value less than 0.05 was considered significant.

\section{Acknowledgments}

This work was supported by grants from the Broad Medical Research Program, by "Premio Giovane Ricercatore" of the Societá Italiana di Medicina Interna, by a grant from the Associazione per le Malattie Infiammatorie Croniche dell'Intestino (A.M.I.C.I. Lombardia) to S. Danese, and by a grant from Ministerio de Educania'n y Ciencia (SAF2005-00280) to M. Sans. The authors thank R. Santangelo and E. Stigliano for technical assistance with mouse experiments and A. Doni for help with confocal imaging and analysis.

Received for publication November 21, 2006, and accepted in revised form March 30, 2007.

Address correspondence to: Silvio Danese, Laboratory of Immunology and Inflammation, Division of Gastroenterology, Istituto Clinico Humanitas-IRCCS in Gastroenterology, Viale Manzoni, Rozzano, Italy. Phone: 011-39-3392318230; Fax: 011-390282244590; E-mail: sdanese@hotmail.com.

Miquel Sans and Stefania Vetrano contributed equally to this work.
1. Fiocchi, C. 1998. Inflammatory bowel disease: etiology and pathogenesis. Gastroenterology. 115:182-205.

2. Podolsky, D.K. 2002. Inflammatory bowel disease. N. Engl. J. Med. 347:417-429.

3. Hatoum, O.A., and Binion, D.G. 2005. The vasculature and inflammatory bowel disease: contribution to pathogenesis and clinical pathology. Inflamm. Bowel Dis. 11:304-313.

4. Fiocchi, C. 1997. Intestinal inflammation: a complex interplay of immune and nonimmune cell interactions. Am. J. Physiol. 273:G769-G775.

5. Fiocchi, C., Ina, K., Danese, S., Leite, A., and Vogel, J.D. 2003. Alterations of the mucosal immune system in IBD mesenchymal and endothelial cells. In Immune mechanisms in inflammatory bowel disease. Landes Bioscience Publishers. New York, New York, USA. $168-176$.

6. Danese, S., and Fiocchi, C. 2006. Etiopathogenesis of inflammatory bowel diseases. World J. Gastroenterol. 12:4807-4812.

7. Toy, L.S., Yio, X.Y., Lin, A., Honig, S., and Mayer, L.
1997. Defective expression of gp180, a novel CD8 ligand on intestinal epithelial cells, in inflammatory bowel disease. J. Clin. Invest. 100:2062-2071.

8. Pucilowska, J.B., Williams, K.L., and Lund, P.K. 2000. Fibrogenesis. IV. Fibrosis and inflammatory bowel disease: cellular mediators and animal models. Am. J. Physiol. Gastrointest. Liver Physiol. 279:G653-G659.

9. Collins, S.M. 1996. The immunomodulation of enteric neuromuscular function: implications for motility and inflammatory disorders. Gastroenterology. 111:1683-1699.

10. Danese, S., De La Motte, C., and Fiocchi, C. 2004 Platelets in inflammatory bowel disease: clinical, pathological and therapeutical implications. Am.J. Gastroenterol. 99:938-945.

11. Girard, J.P., and Springer, T.A. 1995. High endothelial venules (HEVs): specialized endothelium for lymphocyte migration. Immunol. Today. 16:449-457.

12. Granger, D.N., and Kubes, P. 1994. The microcirculation and inflammation: modulation of leu- kocyte-endothelial cell adhesion. J. Leukoc. Biol. 55:662-675.

13. Danese, S., et al. 2006. Angiogenesis as a novel component of inflammatory bowel disease pathogenesis. Gastroenterology. 130:2060-2073.

14. Esmon, C.T. 2001. Protein C anticoagulant pathway and its role in controlling microvascular thrombosis and inflammation. Crit. Care Med. 29:S48-S51; discussion S51-S52.

15. Esmon, C.T. 2002. New mechanisms for vascular control of inflammation mediated by natural anticoagulant proteins. J. Exp. Med. 196:561-564.

16. Esmon, C.T. 2003. The protein C pathway. Chest. 124:26S-32S.

17. Dahlback, B., and Villoutreix, B.O. 2005. The anticoagulant protein C pathway. FEBS Lett. 579:3310-3316.

18. Mosnier, L.O., Zlokovic, B.V., and Griffin, J.H. 2006. The cytoprotective protein C pathway. Blood. 109:3161-3172.

19. Conway, E.M., et al. 2002. The lectin-like domain of 
thrombomodulin confers protection from neutrophil-mediated tissue damage by suppressing adhesion molecule expression via nuclear factor kappaB and mitogen-activated protein kinase pathways. J. Exp. Med. 196:565-577.

20. Abeyama, K., et al. 2005. The $\mathrm{N}$-terminal domain of thrombomodulin sequesters high-mobility groupB1 protein, a novel antiinflammatory mechanism. J. Clin. Invest. 115:1267-1274. doi:10.1172/ JCI200522782.

21. Esmon, C.T. 2004. Structure and functions of the endothelial cell protein $\mathrm{C}$ receptor. Crit. Care Med. 32(5 Suppl.):S298-S301.

22. Iwaki, T., Cruz, D.T., Martin, J.A., and Castellino, F.J. 2005. A cardioprotective role for the endothelial protein $\mathrm{C}$ receptor in lipopolysaccharide-induced endotoxemia in the mouse. Blood. 105:2364-2371.

23. Taylor, F.B., Jr., et al. 1987. Protein C prevents the coagulopathic and lethal effects of Escherichia coli infusion in the baboon. J. Clin. Invest. 79:918-925.

24. Murakami, K., et al. 1997. Activated protein C prevents LPS-induced pulmonary vascular injury by inhibiting cytokine production. Am. J. Physiol. 272:L197-L202

25. Murakami, K., et al. 1996. Activated protein C attenuates endotoxin-induced pulmonary vascular injury by inhibiting activated leukocytes in rats. Blood. 87:642-647.

26. Mizutani, A., Okajima, K., Uchiba, M., and Noguchi, T. 2000. Activated protein C reduces ischemia/ reperfusion-induced renal injury in rats by inhibiting leukocyte activation. Blood. 95:3781-3787.

27. Joyce, D.E., Gelbert, L., Ciaccia, A., DeHoff, B., and Grinnell, B.W. 2001. Gene expression profile of antithrombotic protein c defines new mechanisms modulating inflammation and apoptosis. J. Biol. Chem. 276:11199-11203.

28. Franscini, N., et al. 2004. Gene expression profiling of inflamed human endothelial cells and influence of activated protein C. Circulation. 110:2903-2909.

29. Van der Meeren, A., Vandamme, M., Squiban, C., Gaugler, M.H., and Mouthon, M.A. 2003. Inflammatory reaction and changes in expression of coagulation proteins on lung endothelial cells after totalbody irradiation in mice. Radiat. Res. 160:637-646.

30. Yuda, H., et al. 2004. Activated protein C inhibits bronchial hyperresponsiveness and Th2 cytokine expression in mice. Blood. 103:2196-2204.

31. Terada, Y., et al. 2003. Capillary endothelial thrombomodulin expression and fibrin deposition in rats with continuous and bolus lipopolysaccharide administration. Lab. Invest. 83:1165-1173.

32. Danese, S., et al. 2006. Inflammation and coagulation in inflammatory bowel disease: the clot thickens. Am. J. Gastroenterol. 102:174-186.

33. Faioni, E.M., et al. 2004. Expression of endothelial protein $\mathrm{C}$ receptor and thrombomodulin in the intestinal tissue of patients with inflammatory bowel disease. Crit. Care Med. 32(5 Suppl.):S266-S270.

34. Danese, S., et al. 2003. Platelets trigger a CD40dependent inflammatory response in the microvasculature of inflammatory bowel disease patients. Gastroenterology. 124:1249-1264.

35. Feagan, B.G., et al. 2005. Treatment of ulcerative colitis with a humanized antibody to the alpha4beta7 integrin. N. Engl. J. Med. 352:2499-2507.

36. Podolsky, D.K. 2005. Selective adhesion-molecule therapy and inflammatory bowel disease--a tale of Janus? N. Engl. J. Med. 353:1965-1968.

37. Panes, J., and Granger, D.N. 1998. Leukocyteendothelial cell interactions: molecular mechanisms and implications in gastrointestinal disease. Gastroenterology. 114:1066-1090.

38. Laroux, F.S., and Grisham, M.B. 2001. Immunological basis of inflammatory bowel disease: role of the microcirculation. Microcirculation. 8:283-301.

39. Danese, S., et al. 2005. Starving the inflamed gut: angiogenesis blockade as a novel therapeutic approach to experimental colitis [abstract]. Gastroenterology. 128 (Suppl. 2):A40

40. Spencer, D.M., Veldman, G.M., Banerjee, S., Willis, J., and Levine, A.D. 2002. Distinct inflammatory mechanisms mediate early versus late colitis in mice. Gastroenterology. 122:94-105.

41. Krieglstein, C.F., et al. 2002. Collagen-binding integrin $\alpha 1 \beta 1$ regulates intestinal inflammation in experimental colitis. J. Clin. Invest. 110:1773-1782. doi:10.1172/JCI200215256.

42. Sans, M., et al. 1999. VCAM-1 and ICAM-1 mediate leukocyte-endothelial cell adhesion in rat experimental colitis. Gastroenterology. 116:874-883.

43. Van de Wouwer, M., Collen, D., and Conway, E.M. 2004. Thrombomodulin-protein C-EPCR system: integrated to regulate coagulation and inflammation. Arterioscler. Thromb. Vasc. Biol. 24:1374-1383.

44. Esmon, C.T. 2004. Crosstalk between inflammation and thrombosis. Maturitas. 47:305-314.

45. Okajima, K. 2004. Regulation of inflammatory responses by activated protein $\mathrm{C}$ : the molecular mechanism(s) and therapeutic implications. Clin. Chem. Lab. Med. 42:132-141.

46. Biedermann, B.C. 2001. Vascular endothelium: checkpoint for inflammation and immunity. News Physiol. Sci. 16:84-88.

47. Esmon, C.T. 2003. Inflammation and thrombosis. J. Thromb. Haemost. 1:1343-1348.

48. Frantz, S., Vincent, K.A., Feron, O., and Kelly, R.A. 2005. Innate immunity and angiogenesis. Circ. Res. 96:15-26.

49. Macias, W.L., et al. 2005. New insights into the protein $C$ pathway: potential implications for the biological activities of drotrecogin alfa (activated).
Crit. Care. 9(Suppl. 4):S38-S45.

50. Grinnell, B.W., and Joyce, D. 2001. Recombinant human activated protein C: a system modulator of vascular function for treatment of severe sepsis. Crit. Care Med. 29(7 Suppl.):S53-S60; discussion S60-S61.

51. Nick, J.A., et al. 2004. Recombinant human activated protein $C$ reduces human endotoxin-induced pulmonary inflammation via inhibition of neutrophil chemotaxis. Blood. 104:3878-3885.

52. Schoots, I.G., et al. 2004. Inhibition of coagulation and inflammation by activated protein $\mathrm{C}$ or antithrombin reduces intestinal ischemia/reperfusion injury in rats. Crit. Care Med. 32:1375-1383.

53. Isobe, H., Okajima, K., Harada, N., Liu, W., and Okabe, H. 2004. Activated protein C reduces stressinduced gastric mucosal injury in rats by inhibiting the endothelial cell injury. J. Thromb. Haemost. 2:313-320.

54. Levi, M., Choi, G., Schoots, I., Schultz, M., and van der Poll, T. 2004. Beyond sepsis: activated protein $\mathrm{C}$ and ischemia-reperfusion injury. Crit. Care Med. 32(5 Suppl.):S309-S312.

55. Suzuki, K., et al. 2004. Protective role of activated protein $\mathrm{C}$ in lung and airway remodeling. Crit. Care Med. 32(5 Suppl.):S262-S265.

56. Bernard, G.R., et al. 2001. Efficacy and safety of recombinant human activated protein $\mathrm{C}$ for severe sepsis. N. Engl. J. Med. 344:699-709.

57. Parrillo, J.E. 2005. Severe sepsis and therapy with activated protein C. N. Engl. J. Med. 353:1398-1400.

58. Vogel, J.D., et al. 2004. CD40-mediated immunenonimmune cell interactions induce mucosal fibroblast chemokine leading to T-cell trasmigration. Gastroenterology. 126:63-80.

59. Yan, S.C., et al. 1990. Characterization and novel purification of recombinant human protein $\mathrm{C}$ from three mammalian cell lines. Biotechnology (N.Y.). 8:655-661.

60. Sandusky, G., Berg, D.T., Richardson, M.A., Myers, L., and Grinnell, B.W. 2002. Modulation of thrombomodulin-dependent activation of human protein C through differential expression of endothelial Smads. J. Biol. Chem. 277:49815-49819.

61. Gruber, A., and Griffin, J.H. 1992. Direct detection of activated protein $\mathrm{C}$ in blood from human subjects. Blood. 79:2340-2348.

62. Berg, D.T., et al. 2003. Engineering the proteolytic specificity of activated protein $\mathrm{C}$ improves its pharmacological properties. Proc. Natl. Acad. Sci. U. S. A. 100:4423-4428.

63. Lieberman, B.Y., Fiocchi, C., Youngman, K.R., Sapatnekar, W.K., and Proffitt, M.R. 1988. Interferon gamma production by human intestinal mucosal mononuclear cells. Decreased levels in inflammatory bowel disease. Dig. Dis. Sci. 33:1297-1304. 